\title{
Characterization of fine particles using optomagnetic measurements
}

\author{
Fock, Jeppe; Jonasson, Christian; Johansson, Christer; Hansen, Mikkel Fougt
}

Published in:

Physical Chemistry Chemical Physics

Link to article, DOI:

$10.1039 / \mathrm{c} 6 \mathrm{cp} 08749 \mathrm{c}$

Publication date:

2017

Document Version

Peer reviewed version

Link back to DTU Orbit

Citation (APA):

Fock, J., Jonasson, C., Johansson, C., \& Hansen, M. F. (2017). Characterization of fine particles using optomagnetic measurements. Physical Chemistry Chemical Physics, 19, 8802-8814.

https://doi.org/10.1039/c6cp08749c

\section{General rights}

Copyright and moral rights for the publications made accessible in the public portal are retained by the authors and/or other copyright owners and it is a condition of accessing publications that users recognise and abide by the legal requirements associated with these rights.

- Users may download and print one copy of any publication from the public portal for the purpose of private study or research.

- You may not further distribute the material or use it for any profit-making activity or commercial gain

- You may freely distribute the URL identifying the publication in the public portal

If you believe that this document breaches copyright please contact us providing details, and we will remove access to the work immediately and investigate your claim 


\title{
Journal Name
}

\section{ARTICLE TYPE}

Cite this: DOI: 10.1039/xxxxxxxxxx

Received Date

Accepted Date

DOI: 10.1039/xxxxxxxxxx

www.rsc.org/journalname

\section{Characterization of fine particles using optomagnetic measurements ${ }^{\dagger}$}

\author{
Jeppe Fock, ${ }^{a}$ Christian Jonasson, ${ }^{b}$ Christer Johansson, ${ }^{b}$ and Mikkel Fougt Hansen ${ }^{* a}$
}

\begin{abstract}
The remanent magnetic moment and the hydrodynamic size are important parameters for the synthesis and applications of magnetic nanoparticles (MNPs). We present the theoretical basis for the determination of the remanent magnetic moment and the hydrodynamic size of MNPs with a narrow size distribution by use of optomagnetic measurements. In these, the 2nd harmonic variation of the intensity of light transmitted through an MNP suspension is measured as function of an applied axial oscillating magnetic field. We first show how measurements of the optomagnetic signal magnitude at a low frequency vs. magnetic field amplitude can be used to determine the MNP moment. Subsequently, we use linear response theory to describe the dynamic non-equilibrium response of the MNP suspension at low magnetic field amplitudes and derive a link between optomagnetic measurements and magnetic AC susceptibility measurements. We demonstrate the presented methodology on two samples of commercially available multi-core MNPs. The results compare well with those obtained by dynamic light scattering, AC susceptibility and vibrating sample magnetometry measurements on the same samples when the different weighting of the particle size in the techniques is taken into account. The optomagnetic technique is simple, fast and does not require prior knowledge of the concentration of MNPs and it thus has potential to be used as a routine tool for quality control of MNPs.
\end{abstract}

\section{Introduction}

Magnetic nanoparticle (MNP) suspensions are important for many applications including shock absorbers (magnetorheological fluids), magnetic fluid hyperthermia, magnetic resonance imaging (MRI), and biodetection. ${ }^{1}$

Magnetic nanoparticles can be very complex nanomaterials. Single-core particles consists of a single crystalline and single domain magnetic core surrounded by a shell. In contrast, multi-core nanoparticles feature a particle core containing several such magnetic cores embedded in or encapsulated by, e.g., a polymer. Depending on, e.g., their size, the magnetic cores may be superparamagnetic or magnetically blocked relative to the physical orientation of the MNP. In the following we will focus on the determination of the effective remanent magnetic moment and the

\footnotetext{
a Department of Micro- and Nanotechnology, DTU Nanotech, Bldg. 345B, Technical University of Denmark, DK-2800 Kongens Lyngby, Denmark, Email: jepf@nanotech.dtu.dk, Mikkel.Hansen@nanotech.dtu.dk

${ }^{b}$ Acreo Swedish ICT AB,Box 53071,SE-400 14 Gothenburg, Sweden.

$\dagger$ Electronic Supplementary Information (ESI) available: Sections S1-S3 contain a derivation of the phase-sensitive detection of the response, a calculation of the reference signal and an analytical approximation to the field-dependent equilibrium response. Section S4 presents important relations for the lognormal distribution. Sections S5 and S6 present vibrating sample magnetometry and AC susceptibility data, respectively. Data from figures $2-6$ are included as xls files. See DOI: 10.1039/b000000x/
}

hydrodynamic size of single-core and multi-core MNPs.

These properties are in most cases critical parameters for the performance of the particle suspensions and are moreover highly sensitive to a variation in the conditions during fabrication of the particles. Therefore, measurements of the particle size and magnetic moment are parameters of key importance for the quality control of commercially produced nanoparticle suspensions. In many cases the hydrodynamic particle size is measured batchwise using dynamic light scattering (DLS). However, due to the expensive and time consuming measurements required to measure the magnetic moment, this is often only measured in the R\&D process and is not included in a continuous quality monitoring process during particle production.

The size of nanoparticles can be obtained using a range of methods that each has advantages and disadvantages. ${ }^{2}$ In transmission electron microscopy (TEM), the size of the magnetic cores and particles are estimated from the $2 \mathrm{D}$ projection images. The non-magnetic shell has low contrast compared to iron oxide magnetic cores and particles may further agglomerate during sample preparation. Thus, TEM imaging provides a good method to measure the magnetic core sizes, but it can be difficult to extract the particle size from the images. Atomic force microscopy is also used to measure the size (height) of the particles on a substrate. Both methods provide number-weighted distributions. However, 
they rely on expensive equipment, trained users, they are time consuming, and sample preparation can be difficult.

Several scattering techniques are also used to extract the particle size distribution. From X-ray diffraction (XRD), the mean magnetic core size can be determined from the width of the diffraction peaks. Small-angle X-ray scattering (SAXS) can be used to extract the particle size. ${ }^{3}$ Static light scattering (SLS) and dynamic light scattering (DLS) can be used to extract the mean hydrodynamic particle size and to assess the distribution of hydrodynamic particle sizes. For the optical methods, the nanoparticle suspension has to be very clean because a small amount of dust in the solution can dominate the measurement. Therefore, a filtration step is often applied before the measurement.

AC susceptibility (ACS) measurements can provide information on the hydrodynamic size of magnetic nanoparticles with a remanent magnetic moment. For the simplest case, the Brownian relaxation frequency $\left(f_{\mathrm{B}}\right)$ is found as the peak position of the out-of phase magnetic susceptibility and the size is determined from

$$
f_{\mathrm{B}}=\frac{k_{\mathrm{B}} T}{6 \pi \eta V_{\mathrm{h}}},
$$

where $k_{\mathrm{B}} T$ is the thermal energy, $\eta$ is the dynamic viscosity and $V_{\mathrm{h}}$ is the particle hydrodynamic volume. At low amplitude of the applied magnetic field and at low frequencies, ACS measurements probe the equilibrium initial susceptibility, which is proportional to the square of the particle magnetic moment. Thus, ACS measurements can also be used to extract the mean magnetic moment of the particles using the amplitude of the signal assuming that the particle concentration in the sample is known. ${ }^{4}$ Models that take the size distribution into account can also be applied. In these, the spectra are fitted using the Debye model with a distribution of hydrodynamic volumes or particle core volumes. 5,6 The models can also include Néel relaxation. ${ }^{7}$ It is also possible to extract the mean magnetic moment of the particles from more non-standard ACS measurements of the Brownian relaxation response in a range of transverse applied magnetic fields. ${ }^{8}$ Finally, the magnetic moment can be estimated using vibrating sample magnetometry, or, for single-core particles, from the core size measured by TEM assuming a known magnetization.

Optical measurements on magnetically actuated nanoparticles have been used extensively for biodetection assays ${ }^{9-16}$ as well as for the characterization of the hydrodynamic size of magnetic nanorods. $10,17,18$

We have previously introduced a simple optical method that measures the modulation of light transmitted through an MNP suspension in response to an applied axial oscillating magnetic field, and demonstrated this method for biodetection. ${ }^{12-16}$ The basis for the method is measurements of the magnetic-fieldinduced modulation of the optical extinction of the MNP suspension. To distinguish the method from measurements of traditional magnetooptical effects, it was termed optomagnetic (OM). For a sinusoidal magnetic field variation, it was hypothesized that the complex OM signal is related to the complex ACS signal as ${ }^{13,14}$

$$
V_{2}=\mathrm{i} V_{\mathrm{AC}} \tilde{\chi}^{2},
$$

where $V_{\mathrm{AC}}$ is a real constant describing the scattering properties of the particles in the measurement setup, $\tilde{\chi}=\chi / \chi_{0}$ is the magnetic susceptibility normalized with the DC susceptibility, and $V_{2}$ is the optomagnetic signal measured as the second harmonic of the signal from the photodetector (see Fig. 1c). The relation, although being based on reasonable assumptions, was not derived theoretically.

Here, we present the theoretical basis for OM measurements on MNP suspensions and demonstrate that the OM method can be used to extract both the magnetic moment and hydrodynamic size of magnetic nanoparticles in suspension with a blocked magnetic moment and a comparatively narrow size distribution.

In Section 2 we describe the theories for the equilibrium response and the dynamic response of suspensions of ensembles of identical particles in an applied oscillating magnetic field and we calculate the resulting OM signal. Then, we give the corresponding expressions for particle suspensions with distributions of parameters. In Section 4 we show experimental examples where the measurement technique is applied to determine the remanent magnetic moment (Section 4.1) and the hydrodynamic size (Section 4.2) of two multi-core nanoparticle samples. We will show that OM method provides information comparable to ACS, but the measurement is fast (less than $10 \mathrm{~min}$ ), it does not require prior knowledge of the particle concentration to determine the particle magnetic moment and it can be made at low cost. Further, there are subtle differences between the two techniques that will be elaborated upon below.

\section{Theory}

In Section 2.1 we first describe the basic principle of the OM measurement as performed by us on a suspension of MNPs with a remanent magnetic moment. Then, in Section 2.2 we develop an equilibrium model that can be used to determine the remanent magnetic moment from measurements of the low-frequency response vs. magnetic field amplitude. Finally, in Section 4.2.1 we calculate the linear response of the particles to obtain the dynamics of relaxing magnetic particles in a magnetic field and we derive the expression for the dynamic OM signal.

\subsection{The optomagnetic method}

The technique relies on synchronized measurements of the intensity of light transmitted through a suspension of MNPs, $I(t)$, in response to an oscillating applied magnetic field,

$$
B(t)=B_{0} \sin (2 \pi f t) .
$$

The sinusoidal magnetic field variation is chosen to make the analysis consistent with the detection electronics used in our initial work and because it has the convenient feature that the field is zero at time $t=0$. Fig. 1 shows a schematic of the behavior during a period of magnetic excitation at a frequency $f \ll f_{\mathrm{B}}$. When $B(t) \approx 0$ the particles are randomly orientated. However, at finite magnetic fields the particles partly align their magnetic moments with the field. When the particles have an optical anisotropy, which is correlated to the magnetic orientation, an increase (or decrease) in light intensity is observed at finite (negative as well 


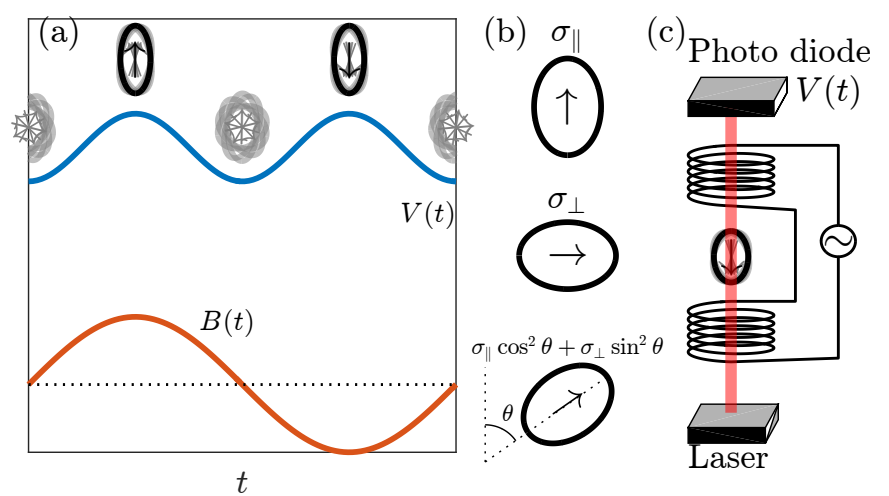

Fig. 1 (a) Schematic of one period of the magnetic field excitation (red) and the corresponding modulation of intensity of transmitted light (blue). The particle orientations are indicated as ellipses with an arrow showing the magnetic moment. (b) The extinction cross-sections of particles oriented parallel to, perpendicular to and at an angle $\theta$ to the light direction. (c) Schematic of the setup. The photodetector signal $V(t)$ measures the intensity of light transmitted through a suspension of magnetic particles. The light intensity is modulated by an external magnetic field generated from two coils controlled by a current source.

as positive) magnetic fields.

To develop a model for the OM response, we assume that the particles have extinction cross sections $\sigma_{\|}$and $\sigma_{\perp}$ when they are aligned parallel and perpendicular to the light path, respectively (Fig. 1b). Thus, $\sigma_{\|}$is the extinction cross section parallel to the magnetic moment and $\sigma_{\perp}$ is the average extinction cross section perpendicular to the moment direction. The normalized intensity of transmitted light for a homogeneous suspension of MNPs is then ${ }^{19}$

$$
T(t)=\frac{I(t)}{I_{0}}=\exp \left[-n z\left(\left(\sigma_{\|}-\sigma_{\perp}\right) \overline{e_{z}^{2}}(t)+\sigma_{\perp}\right)\right] .
$$

Here, $I_{0}$ and $I(t)$ are the intensities of the incoming and transmitted light, respectively, $n$ is the MNP number concentration and $z$ is the optical path length. $\overline{e_{z}^{2}}(t)$ is the time-dependent ensemble average of $\cos ^{2} \theta$ where $\theta$ is the angle between the optical anisotropy of the particle and the light path. For randomly oriented particles, $\overline{e_{z}^{2}}=1 / 3$.

$T(t)$ is independent of the polarization of the light because the magnetic field is applied along the light path. Consequently, the particle orientations perpendicular to the light path are random and no dependence on light polarization is observed. ${ }^{14}$ We therefore perform measurements using unpolarized light.

In practice we measure the photodetector voltage $V(t)=$ $Q I_{0} T(t)$, where $Q$ is the quantum efficiency of the photodetector. Thus, the signal from a suspension of randomly oriented particles is

$$
V_{\text {ref }}=Q I_{0} \exp \left[-\frac{1}{3} n z\left(2 \sigma_{\perp}+\sigma_{\|}\right)\right]
$$

To correct for possible variations in $I_{0}$, we normalize the signal by $V_{\text {ref }}$. In the limit of thin samples and small $\Delta \sigma=\sigma_{\|}-\sigma_{\perp}$ where $n z \Delta \sigma \ll 1$, the signal becomes

$$
\frac{V(t)}{V_{\mathrm{ref}}}=1-n z \Delta \sigma\left(\overline{e_{z}^{2}}(t)-\frac{1}{3}\right) .
$$

The signal is measured using phase-sensitive detection (fast Fourier transformation (FFT) of the time response or lock-inbased detection). We can write the normalized $p$ th harmonic complex signal, $V_{p}(f)=V_{p}^{\prime}(f)+\mathrm{i} V_{p}^{\prime \prime}(f)$, with respect to the magnetic field modulation at frequency $f$ as

$$
\frac{V_{p}(f)}{V_{\text {ref }}}=-n z \Delta \sigma 2 \mathrm{i} c_{p}(f), p \geq 1
$$

and

$$
\frac{V_{0}}{V_{\text {ref }}}=1-n z \Delta \sigma\left(c_{0}-\frac{1}{3}\right)
$$

with

$$
c_{p}(f)=f \int_{0}^{1 / f} \overline{e_{z}^{2}}(t) \exp (-2 \pi \mathrm{i} p f t) \mathrm{d} t .
$$

Here $c_{p}$ is the complex Fourier coefficient of the $p$ th harmonic of $\overline{e_{z}^{2}}(t)$, and $2 \mathrm{i} c_{p}$ is the corresponding FFT signal response to a sine reference signal for $p \geq 0$ (see Section $\mathrm{S} 1^{\dagger}$ ).

From Eq. (8) it is observed that the mean signal, $V_{0}$, is equal to $V_{\text {ref }}$ for randomly oriented particles for which $c_{0}=1 / 3$. In an applied magnetic field $c_{0} \neq 1 / 3$ and thus $V_{\text {ref }}$ can no longer be assumed to be equal to $V_{0}$. Writing the signal as a Taylor series in terms of the magnetic field excitation, $V(t)=V_{\text {ref }}+$ $\sum_{p=1}^{\infty} S_{p} \sin ^{p}(2 \pi f t)$, we find that all even powers ( $\left.2 p\right)$ contribute not only to a signal in the $2 p$ th harmonic signal, $V_{2 p}$, but also to the lower even harmonics (see Section $\mathrm{S}^{\dagger}{ }^{\dagger}$ ), and we find that

$$
V_{\mathrm{ref}}=\sum_{p=0}^{N} V_{2 p}
$$

For the analysis of measurements, $N$ should be chosen large enough to ensure that $V_{2 N}$ is lower than the measurement noise.

Comparing the OM signal, Eq. (7), with the signal from AC susceptibility measurements, i.e., the ensemble average of the projection of the magnetic moment of the particles along the applied magnetic field, it is clear that the two methods are closely related. Below, this relation will be investigated further in the derivation of the equilibrium model and in derivation of the dynamic OM response.

\subsection{Equilibrium model}

The magnetic nanoparticles have a remanent magnetic moment $\mathbf{m}$, which is assumed to be independent of the applied magnetic field. Further, assuming that the particles have an optical anisotropy, which is aligned along the remanent magnetic moment, $\overline{e_{z}^{2}}$ can be found analytically in equilibrium using Boltzmann statistics. The probability for the magnetic moment to form an angle $\theta$ to a magnetic field $\mathbf{B}$ is proportional to $\exp (\beta \cos \theta)$, where $\beta=B \cdot m /\left(k_{\mathrm{B}} T\right)$ is the ratio between the magnetic and thermal energies. Below, we denote thermal equilibrium values by $\langle\cdots\rangle$ and ensemble averages by $\cdots$. For a magnetic field applied 


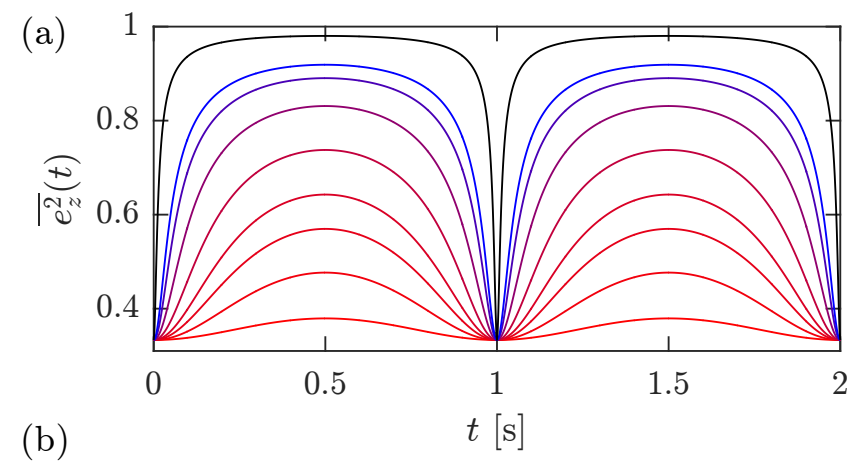

(b)

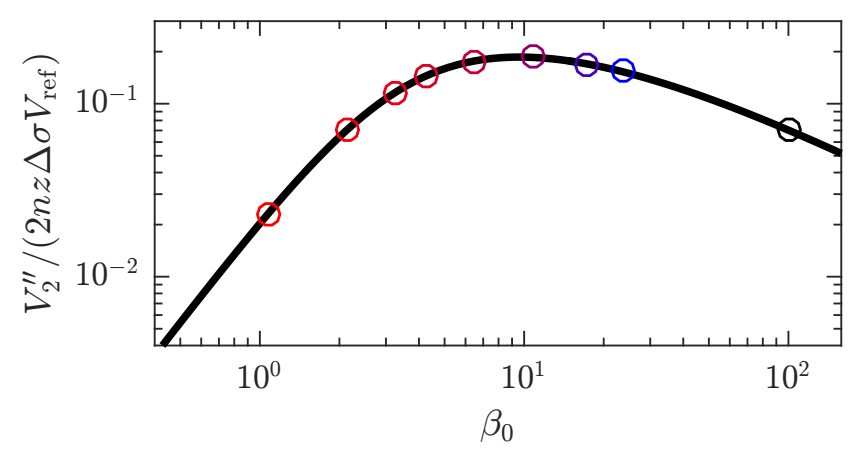

Fig. 2 (a) Optomagnetic response calculated vs. time for $\beta_{0}=1.1,2.2$, $3.3,4.4,6.5,11,18,21,100$. (b) Numerically calculated imaginary 2nd harmonic of the equilibrium response, $\overline{e_{z}^{2}}(t)$. The circles correspond to the curves shown in (a).

along the light path (Fig. 1), we obtain

$$
\begin{aligned}
& \left\langle\overline{e_{z}}\right\rangle=\operatorname{coth}(\beta)-\frac{1}{\beta}=\mathscr{L}(\beta) \simeq \frac{\beta}{3}+\cdots \\
& \left\langle\overline{e_{z}^{2}}\right\rangle=1-\frac{2}{\beta} \mathscr{L}(\beta) \simeq \frac{1}{3}+\frac{2 \beta^{2}}{45}+\cdots
\end{aligned}
$$

where $\mathscr{L}(\beta)$ is the Langevin function, and the expressions after $\simeq$ are Taylor expansions to second order. The Taylor series converges for $\beta<\pi$. For small $\beta$, the Taylor expansion enables us to relate $\left\langle\overline{e_{z}^{2}}\right\rangle$ to $\left\langle\overline{e_{z}}\right\rangle$ as

$$
\left\langle\overline{e_{z}^{2}}\right\rangle \simeq \frac{1}{3}+\frac{2}{5}\left\langle\overline{e_{z}}\right\rangle^{2}
$$

Fig. 2a shows the equilibrium response calculated during one pe$\operatorname{riod}$ of $\beta(t)=\beta_{0} \sin (2 \pi f t)$ from Eq. (12) with $f=0.5 \mathrm{~Hz}$ and the indicated values of $\beta_{0}$. As a sinusoidal magnetic field excitation is used and the signal only depends on the absolute value of the magnetic field, the signal, which is in phase with the excitation is measured in $V_{2}^{\prime \prime}$ (see Section $\mathrm{S} 1^{\dagger}$ ). Fig. $2 \mathrm{~b}$ shows the normalized imaginary value of the second harmonic signal, $V_{2}^{\prime \prime} /\left(2 n z \Delta \sigma V_{\text {ref }}\right)=2 \mathrm{i} c_{2}\left(\beta_{0}\right)$, calculated numerically for increasing values of $\beta_{0}$. This signal shows a maximum at $\beta_{0} \simeq 9.4$. This maximum is thus a characteristic feature related to the magnitude of the magnetic moment.

Experimentally, the field-dependent equilibrium response can be obtained by measuring $V_{2}^{\prime \prime}$ vs. magnetic field amplitude at a frequency, which is low enough to ensure that the response is frequency-independent and in phase with the magnetic field excitation. The magnetic moment can be obtained by analyzing the measurements in terms of the magnitude of

$$
\frac{V_{2}\left(B_{0}\right)}{V_{\text {ref }}}=-n z \Delta \sigma 2 \mathrm{ic} c_{2}\left(\frac{B_{0} \cdot m}{k_{\mathrm{B}} T}\right) .
$$

Generally, $c_{2}\left(\beta_{0}\right)$ can be evaluated numerically. In Section $\mathrm{S}^{\dagger}$, we present an analytical approximation to $c_{2}\left(\beta_{0}\right)$, which can be used in a fitting routine.

\subsection{Non-equilibrium model}

The dynamic magnetic response of MNPs in an oscillating magnetic field can for small fields be calculated using linear response theory.

When a magnetic field is applied to a suspension of MNPs, they will rotate to align their moments along the field direction. For low magnetic fields, the characteristic time for this process is the rotational Brownian relaxation time $\tau_{\mathrm{B}}=1 /\left(2 \pi f_{\mathrm{B}}\right)$ with $f_{\mathrm{B}}$ given by Eq. (1). The differential equation governing the approach to equilibrium is

$$
\frac{\mathrm{d} \overline{e_{z}}}{\mathrm{~d} t}=-\frac{1}{\tau_{\mathrm{B}}}\left(\overline{e_{z}}(t)-b(t)\right)
$$

where

$$
b(t)=\left\langle\overline{e_{z}}\right\rangle=\beta(t) / 3
$$

is the equilibrium value of $\cos \theta$ in the low-field limit as given by Eq. (11). Taking the Fourier transform and rearranging we obtain

$$
\overline{e_{z}}(\xi)=\frac{B(\xi)}{1+2 \pi \mathrm{i} \xi \tau_{\mathrm{B}}} .
$$

where $\xi$ is the frequency and $B(\xi)$ is the Fourier transform of $b(t)$. For $b(t)=b_{0} \sin (2 \pi f t)$ we have

$$
B(\xi)=b_{0}(\delta(\xi-f)-\delta(\xi+f)) / 2 \mathrm{i} .
$$

The magnetic susceptibility fulfills $H_{0} \chi(f)=n\langle m \cos \theta\rangle(f)$. Using Eq. (18) and the relation between $\tau_{\mathrm{B}}$ and $f_{\mathrm{B}}$ we obtain the wellknown Debye model

$$
\chi(f)=\frac{\chi_{0}}{1+\mathrm{i} f / f_{\mathrm{B}}}
$$

with $\chi_{0}=n \mu_{0} m^{2} /\left(3 k_{\mathrm{B}} T\right)$.

The low-field approximation, Eq. (13), relates $\overline{e_{z}^{2}}$ to $\overline{e_{z}}$ at low frequencies where $\overline{e_{z}^{2}}=\left\langle\overline{e_{z}^{2}}\right\rangle$ and $\overline{e_{z}}=\left\langle\overline{e_{z}}\right\rangle$ (thermal equilibrium). Making the simplifying assumption that this relation also holds for the dynamic ensemble averages, i.e., that $\overline{e_{z}^{2}} \simeq \frac{1}{3}+\frac{2}{5} \bar{e}_{z}^{2}$, and performing a time differentiation, we can write

$$
\frac{\mathrm{d} \overline{e_{z}^{2}}}{\mathrm{~d} t} \simeq \frac{2}{5} \frac{\mathrm{d}\left(\overline{e_{z}}\right)}{\mathrm{d} t}=\frac{4}{5} \overline{e_{z}} \frac{\mathrm{d} \overline{e_{z}}}{\mathrm{~d} t} .
$$

Taking the Fourier transform and inserting $\overline{e_{z}}(\xi)$ from Eq. (17), 
we obtain

$$
\begin{aligned}
\mathrm{i} 2 \pi \xi \overline{e_{z}^{2}}(\xi) & =\frac{4}{5} \int \frac{B\left(\xi-\xi^{\prime}\right)}{1+\mathrm{i}\left(\xi-\xi^{\prime}\right) / f_{\mathrm{B}}} 2 \pi \mathrm{i} \xi^{\prime} \frac{B\left(\xi^{\prime}\right)}{1+\mathrm{i}\left(\xi^{\prime}\right) / f_{\mathrm{B}}} \mathrm{d} \xi^{\prime} \\
\overline{e_{z}^{2}}(\xi) & =-\frac{b_{0}^{2}}{5} \frac{\mathrm{i} f}{\mathrm{i} \xi} \frac{1}{1 \pm \mathrm{i} f / f_{\mathrm{B}}} \frac{\delta(\xi \mp 2 f)-\delta(\xi)}{1+\mathrm{i}(\xi \mp f) / f_{\mathrm{B}}} .
\end{aligned}
$$

In the last step, we inserted $B(\xi)$ from Eq. (18) and evaluated the convolution integral. The second harmonic response obtained by inserting $\xi=2 f$ in Eq. (22) is

$$
2 \mathrm{i} c_{2}(f)=-\frac{\mathrm{i} b_{0}^{2}}{5}\left(\frac{1}{1+\mathrm{i} f / f_{\mathrm{B}}}\right)^{2} .
$$

Inserting the definition of $b_{0}$, we can write the low-field 2nd harmonic optomagnetic signal as

$$
\frac{V_{2}(f)}{V_{\text {ref }}}=\frac{\mathrm{i} n z \Delta \sigma}{45}\left(\frac{B_{0} m}{k_{\mathrm{B}} T}\right)^{2}\left(\frac{1}{1+\mathrm{i} f / f_{\mathrm{B}}}\right)^{2} .
$$

Eq. (24) has the same frequency dependence as Eq. (2). Thus, we can identify $V_{\mathrm{AC}}$ in Eq. (2) as

$$
V_{\mathrm{AC}}=\frac{n z \Delta \sigma}{45}\left(\frac{B_{0} m}{k_{\mathrm{B}} T}\right)^{2} V_{\text {ref }} .
$$

It is noteworthy for the employed experimental geometry with the magnetic field applied along the light path that $V_{\mathrm{AC}}$ has the same sign as $\Delta \sigma=\sigma_{\|}-\sigma_{\perp}$. Thus, when $V_{\mathrm{AC}}<0$ we can deduce that $\sigma_{\|}<\sigma_{\perp}$, i.e., more light will be transmitted when the particle magnetic moments are aligned along the light path (the magnetic field direction) than when they are randomly oriented.

The black curves in Fig. 3 show the Debye model for the complex magnetic susceptibility (Fig. 3a-b) and the model for the complex optomagnetic signal, $V_{2}=V_{2}^{\prime}+\mathrm{i} V_{2}^{\prime \prime}$ (Fig. 3c-d) calculated using Eqs. (19) and (24) and normalized with $\chi_{0}$ and $V_{\mathrm{AC}}$ respectively. The frequency axis is normalized with the (median) Brownian relaxation frequency, $f_{\mathrm{Bm}}$.

The in-phase susceptibility, $\chi^{\prime}$, shows a plateau at $f \ll f_{\mathrm{B}}$ and, in the simple Debye model, it drops to zero for $f \gg f_{\mathrm{B}}$ when the particles can no longer follow the field changes. A peak in the out-of-phase susceptibility, $\chi^{\prime \prime}$, is observed at $f=f_{\mathrm{B}}$, which using Eq. (1) can be used to extract the particle size.

The black curve in Fig. 3d shows the imaginary part of the optomagnetic signal, $V_{2}^{\prime \prime}$. This signal is observed to change sign at a frequency $f_{V_{2}^{\prime \prime}=0}$ and for the black curve in Fig. 3d (and only that), it is observed that $f_{V_{2}^{\prime \prime}=0}=f_{\mathrm{B}}$. The black curve in Fig. 3c shows the real part of the optomagnetic signal, $V_{2}^{\prime}$. This signal is observed to display a peak at a frequency $f_{V_{2}^{\prime} \text {,peak }}$ and for the black curve, it is found that $f_{V_{2}^{\prime} \text {,peak }}=f_{\mathrm{B}} / \sqrt{3}$. Thus, both $V_{2}^{\prime}$ and $V_{2}^{\prime \prime}$ can be used to extract the Brownian relaxation frequency and consequently also the hydrodynamic size.

Finally, we note that the above derivations were performed for a sinusoidal magnetic field excitation. In Section $\mathrm{S}^{\dagger}$ we present the corresponding results for a cosine magnetic field excitation.
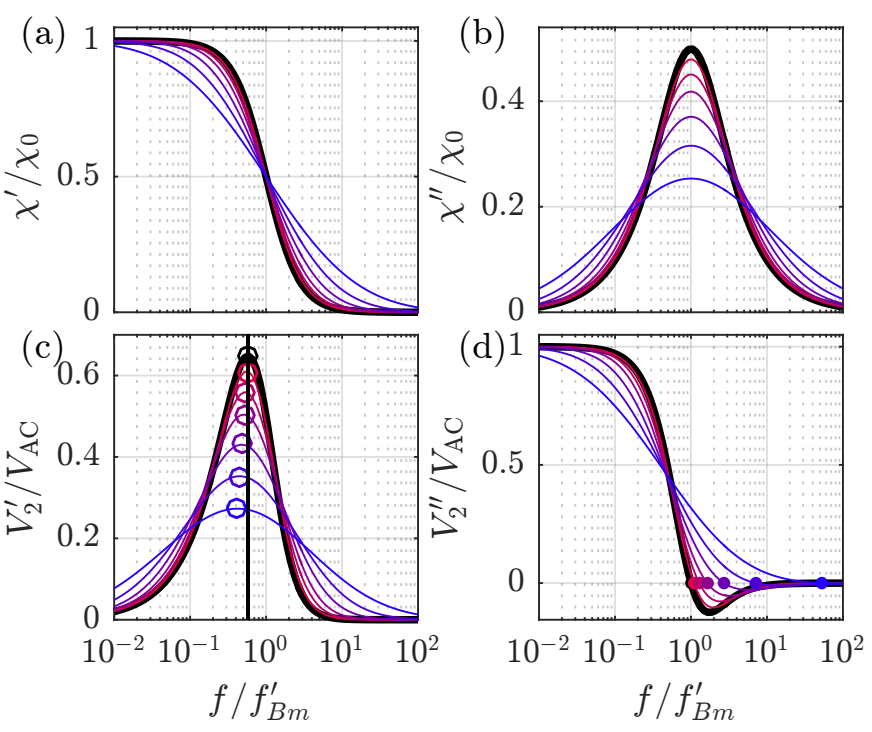

Fig. 3 Calculated magnetic and optomagnetic response vs. normalized frequency for lognormal distributions of Brownian relaxation frequencies with median value $f_{\mathrm{Bm}}^{\prime}$ and $\sigma_{f_{\mathrm{B}}}=0.05,0.1,0.3,0.5,0.7,1,1.4$ and 2 . (a) and (b) show the normalized in-phase and out-of-phase magnetic susceptibility, respectively. (c) and (d) show the normalized real and imaginary parts of the 2 nd harmonic optomagnetic response. In (c) the vertical line indicates $f_{\mathrm{Bm}} / \sqrt{3}$ and the circles indicate the peak positions $\left(f_{V_{2}^{\prime} \text {,peak }}\right)$. In (d) the filled circles indicate the zero crossings $\left(f_{V_{2}^{\prime \prime}=0}\right)$.

\subsection{Distributions of parameters}

The above considerations are only valid for an ensemble of particles with identical properties. However, $V_{2}$ depends on the size, shape and magnetic moment of the particles. Thus, when the distributions of these parameters are not sufficiently narrow, the magnetic field-dependent equilibrium signal, Eq. (14), and the low-field dynamic signal, Eq. (24), have to be integrated over the distribution of parameters. In the most general form, the signals are given by

$V_{2}\left(B_{0}\right)=-n z \iiint V_{\text {ref }}\left(\sigma_{\|}, \sigma_{\perp}\right) \Delta \sigma\left[2 \mathrm{i} c_{2}\left(\frac{B_{0} m}{k_{\mathrm{B}} T}\right)\right] p\left(m, \sigma_{\|}, \sigma_{\perp}\right) \mathrm{d} m \mathrm{~d} \sigma_{\|} \mathrm{d} \sigma_{\perp}$

$V_{2}(f)=\iiint \int \frac{\mathrm{i} n z \Delta \sigma}{45}\left(\frac{B_{0} m}{k_{\mathrm{B}} T}\right)^{2}\left(\frac{1}{1+\mathrm{i} f / f_{\mathrm{B}}}\right)^{2} p\left(f_{\mathrm{B}}, m, \sigma_{\|}, \sigma_{\perp}\right) \mathrm{d} m \mathrm{~d} f_{\mathrm{B}} \mathrm{d} \sigma_{\|} \mathrm{d} \sigma_{\perp}$.

Here, $p\left(f_{\mathrm{B}}, m, \sigma_{\|}, \sigma_{\perp}\right)$ is a distribution describing the number fraction of particles with a Brownian relaxation frequency between $f_{\mathrm{B}}$ and $f_{\mathrm{B}}+\mathrm{d} f_{\mathrm{B}}$, a magnetic moment between $m$ and $m+\mathrm{d} m$, and extinction cross-sections between $\sigma_{\|, \perp}$ and $\sigma_{\|, \perp}+\mathrm{d} \sigma_{\|, \perp}$. The distribution in Eq. (26) is independent of $f_{\mathrm{B}}$ because the measurement is performed at equilibrium $\left(f \ll f_{\mathrm{B}}\right)$.

The distribution, $p(\cdot)$, can be very complex because the parameters may depend both on the size and shape of the particles. $m$ and $\sigma_{\|, \perp}$ both depend on the particle core size and shape and $f_{\mathrm{B}}$ is inversely proportional to the hydrodynamic volume, which again may be correlated to the particle core size. Thus, the correlations depend on the intrinsic structure and morphology of the particles and detailed knowledge on these correlations on a single particle level is required to perform the most general analysis. This knowledge is most often not available. Below we therefore 
make simplifying assumptions and consider two idealized cases for single-core particles and multi-core particles.

We defined a single-core particle as a particle containing a single magnetic core surrounded by a non-magnetic shell. To model a multi-core particle, we will assume that it contains multiple magnetic cores that are randomly oriented with respect to each other within a non-magnetic matrix. We will further assume that the magnetic cores are distributed with constant density within a particle volume that we refer to as the particle core. This volume may be surrounded by a non-magnetic shell. Below, we will consider particles with a nearly spherical shape such that the rotational Brownian relaxation response and hydrodynamic size is well described by that of a sphere. Thus, we assume that the shape of the particle (or particle core) only comes into play via the optical anisotropy. Most commercial nanoparticle samples have a, perhaps irregular, shape with aspect ratios close to one and thus fall into this category. Other classes of particles, such as needle-shaped particles, require a separate analysis to include the effect of the particle shape, e.g., on the Brownian relaxation time via inclusion of an appropriate Perrin rotational friction factor and in some cases, the optical extinction factors can also be described analytically. ${ }^{17}$ For elongated particles, a field-induced magnetic moment may significantly influence the particle dynamics via shape anisotropy. In the present work, however, where the particles have a nearly spherical shape ${ }^{20}$ and where only comparatively weak magnetic fields are applied, this effect is found to be negligible. Further, it is noted that an induced magnetic moment has no effect on the optomagnetic signal unless it influences the orientation of a particle. In the following we assume that the (nearly spherical) shape of the particle is neither correlated with the Brownian relaxation frequency nor the magnitude of the magnetic moment.

To relate the hydrodynamic volume, extinction properties and the magnetic moment to the volume of the particle core, $V_{\mathrm{c}}$, the dependence is assumed to follow a power law as

$$
\begin{gathered}
V_{\mathrm{h}}=\widetilde{V}_{\mathrm{h}}\left(\frac{V_{\mathrm{c}}}{\widetilde{V}_{\mathrm{c}}}\right)^{n_{\mathrm{h}}} \\
\sigma_{\|, \perp}=\widetilde{\sigma}_{\|, \perp}\left(\frac{V_{\mathrm{c}}}{\widetilde{V}_{\mathrm{c}}}\right)^{n_{\sigma \|, \perp}} \\
m=\widetilde{m}\left(\frac{V_{\mathrm{c}}}{\widetilde{V}_{\mathrm{c}}}\right)^{n_{m}}
\end{gathered}
$$

where $\widetilde{V}_{\mathrm{h}}, \widetilde{\sigma}_{\|, \perp}$ and $\widetilde{m}$ are the hydrodynamic volume, extinction properties for the $\|$ and $\perp$ orientations and the moment for $V_{\mathrm{c}}=$ $\widetilde{V}_{\mathrm{c}}$ and $n_{\mathrm{h}}, n_{\sigma \|, \perp}$ and $n_{m}$ are the exponents describing the power dependence.

If the thickness of the non-magnetic shell, $t_{s}$, is large compared to the size of the core, the hydrodynamic volume is expected to be uncorrelated with the core volume $\left(n_{\mathrm{h}}=0\right)$. On the other hand, if $t_{s}$ is small compared to the particle core size, the hydrodynamic volume is assumed to be proportional to the particle core volume $\left(n_{\mathrm{h}}=1\right)$.

The extinction properties will be dominated by the particle core because the particle core will generally have a significantly larger
Table 1 Scaling exponents for particle core volume $\left(x=\widetilde{x} V_{\mathrm{c}}^{n_{x}}\right)$ describing contributions to optical extinction from absorption and scattering $\left(n_{\sigma \|, \perp}\right)$, the magnetic moment $\left(n_{m}\right)$ and the hydrodynamic volume $\left(n_{\mathrm{h}}\right)$ for idealized single-core and multi-core particles approximated as spheres.

Abs
Particle type
Single-core

refractive index than the surrounding liquid, whereas the nonmagnetic shell will have a refractive index similar to that of water. The extinction cross sections depend on both the absorption and scattering of the particles. For non-spherical shapes, the extinction properties can be very complex. In the following we will describe the size-dependence for sphere-like objects with an optical anisotropy along the remanent magnetic moment. We will consider this to be due to a particle core, which is slightly elongated along the magnetic moment.

The absorption scales with the volume, $V_{\mathrm{c}}$, of the particle core. As a first approximation, shadowing will cause particle orientations with a smaller geometric cross-sectional area to have a lower absorption, i.e., $\sigma_{\|}<\sigma_{\perp}$. In metallic particles, surface plasmons may however complicate this picture.

For particles with sizes well below the wavelength of the light, the scattering is proportional to $V_{\mathrm{c}}^{2}$ (Rayleigh scattering). For particles with dimensions comparable to the wavelength of the light, the scattering is angle-dependent and no longer follows a simple dependence on $V_{\mathrm{c}}$ and it can be described by the RayleighGans-Debye approximation. ${ }^{21}$ For a limited size range, the sizedependent scattering approximately follows a power dependence on $V_{\mathrm{c}}$ with $n_{\sigma}$ between 0 and 2 .

It have been shown that the magnetic moment of single-core particles scales with the volume $\left(n_{m}=1\right)$ whereas the moment of multi-core particles consisting of many crystallites scales with the square root of the volume $\left(n_{m}=1 / 2\right) .{ }^{4}$ Table 1 summarizes the above weighting exponents for single-core and multi-core particles.

The distributions, $p\left(m, \boldsymbol{\sigma}_{\|}, \boldsymbol{\sigma}_{\perp}\right)$ and $p\left(f_{\mathrm{B}}, m, \boldsymbol{\sigma}_{\|}, \boldsymbol{\sigma}_{\perp}\right)$ can be simplified to distributions only in moments and in relaxation frequencies, respectively, assuming that the parameters are related according to Eqs. (28)-(30). For narrow size distributions, we will assume that the extinction properties, $\sigma_{\|}$and $\sigma_{\perp}$, have similar size dependencies such that $n_{\sigma_{\|}}=n_{\sigma_{\perp}} \equiv n_{\sigma}$. In this case, Eqs. (26) and (27) can be simplified to

$$
\begin{gathered}
V_{2}\left(B_{0}\right)=-n z \int \widetilde{V}_{\mathrm{ref}} \Delta \widetilde{\sigma}\left[2 \mathrm{i} c_{2}\left(\frac{B_{0} m}{k_{\mathrm{B}} T}\right)\right]\left(\frac{m}{\bar{m}}\right)^{\frac{n_{\sigma}}{n_{m}}} p(m) \mathrm{d} m \\
V_{2}(f)=\int \mathrm{i} \widetilde{V}_{\mathrm{AC}}\left(\frac{1}{1+\mathrm{i} f / f_{\mathrm{B}}}\right)^{2}\left(\frac{f_{\mathrm{B}}}{\widetilde{f}_{\mathrm{B}}}\right)^{-\frac{n_{\sigma}+2 n_{m}}{n_{\mathrm{h}}}} p\left(f_{\mathrm{B}}\right) \mathrm{d} f_{\mathrm{B}}
\end{gathered}
$$


with

$$
\begin{aligned}
& \widetilde{V}_{\text {ref }}=Q I_{0} \exp \left[-n z \frac{2 \widetilde{\sigma_{\perp}}+\widetilde{\sigma_{\|}}}{3}\left(\frac{m}{\widetilde{m}}\right)^{\frac{n_{\sigma}}{n_{m}}}\right] \\
& \widetilde{V}_{\mathrm{AC}}=-\frac{n z \Delta \widetilde{\sigma} B_{0}^{2} \widetilde{m}^{2}}{45\left(k_{\mathrm{B}} T\right)^{2}} Q I_{0} \exp \left[-n z \frac{2 \widetilde{\sigma_{\perp}}+\widetilde{\sigma_{\|}}}{3}\left(\frac{f_{\mathrm{B}}}{\widetilde{f}_{\mathrm{B}}}\right)^{-\frac{n \sigma}{n_{\mathrm{h}}}}\right]
\end{aligned}
$$

and $\Delta \widetilde{\sigma}=\widetilde{\sigma}_{\|}-\widetilde{\sigma}_{\perp}$ and $\widetilde{f_{\mathrm{B}}}=k_{\mathrm{B}} T /\left(6 \pi \eta \widetilde{V}_{\mathrm{h}}\right)$.

In Eqs. (32) and (34) a correlation between the particle core size and the hydrodynamic size is assumed, $n_{\mathrm{h}} \neq 0$. If no such correlation exists, the integration over $m$ and $\sigma_{\|, \perp}$ will be independent of $f_{\mathrm{B}}\left(n_{\sigma}=n_{m}=0\right)$ and $\widetilde{V}_{\mathrm{AC}}=V_{\mathrm{AC}}$ can be treated as a fitting parameter.

For thin nanoparticle samples with sufficiently narrow size and shape distributions, $\widetilde{V}_{\text {ref }}$ and consequently also $\widetilde{V}_{\text {AC }}$ can be considered constant and may therefore be treated as fitting parameters. Consequently the calculation of the equilibrium signal in Eq. (31) and the low-field dynamic signal in Eq. (32) are simple integrations of the single particle signal over the relevant distribution with exponents of the weighting factors of $n_{\sigma} / n_{m}$ and $-\left(n_{\sigma}+2 n_{m}\right) / n_{\mathrm{h}}$, respectively.

\subsubsection{Lognormal distribution}

A lognormal distribution weighted by a power function is also a lognormal distribution but with a scaled median (see Section S4. $\left.4^{\dagger}\right)$. Letting $p_{\ln }(x)$ denote a number-weighted distribution of magnetic moments or hydrodynamic sizes, the weighted distributions in Eqs. (31) and (32) can be written as

$$
p_{\ln }^{\prime}(x)=x^{n} p_{\ln }(x)
$$

The analysis of the data in terms of $p_{\ln }^{\prime}(x)$ results in the median values $m_{\mathrm{m}}^{\prime}$ and $f_{\mathrm{Bm}}^{\prime}$ and widths $\sigma_{m}$ and $\sigma_{f_{\mathrm{B}}}$. The parameters for the number-weighted lognormal distributions, $p_{\ln }(x)$, can subsequently be obtained by scaling the median values according to

$$
\begin{aligned}
m_{\mathrm{m}} & =\exp \left(-\frac{n_{\sigma}}{n_{m}} \sigma_{m}^{2}\right) m_{\mathrm{m}}^{\prime} \\
f_{\mathrm{Bm}} & =\exp \left(\frac{n_{\sigma}+2 n_{m}}{n_{\mathrm{h}}} \sigma_{f_{\mathrm{B}}}^{2}\right) f_{\mathrm{Bm}}^{\prime}
\end{aligned}
$$

Thus $p_{\ln }^{\prime}(x)$ can describe data with power law weighted lognormal distributions of $f_{\mathrm{B}}$ or $m$ for any value of the exponent in the power law. Therefore, a good starting point is to fit the data using a lognormal distribution with no additional power-law weighting (i.e., to use $p_{\ln }^{\prime}(x)$ during fitting). The median values of the numberweighted distribution can then be obtained using Eqs. (36) and (37)

Moreover, the mean value $\bar{g}$ of a lognormal distributed parameter $g$ with median value $g_{\mathrm{m}}$ and distribution width $\sigma_{g}$ is

$$
\bar{g}=g_{\mathrm{m}} \exp \left(\frac{1}{2} \sigma_{g}^{2}\right)
$$

The particle size distribution is often considered to be lognormal. Consequently, it is of interest to calculate model spectra for

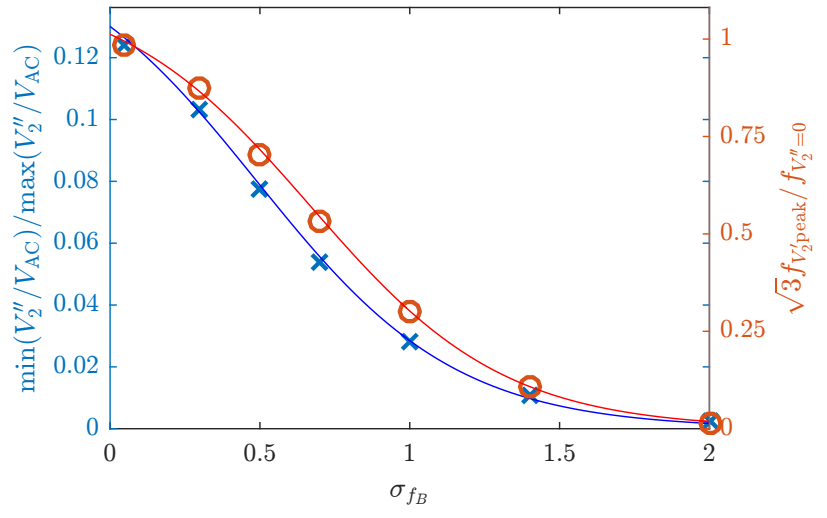

Fig. 4 The ratio between minimum and maximum of $V_{2}^{\prime \prime}$ (left axis), and ratio between peak position and zero crossing (right axis) versus the width of a lognormal distribution, $\sigma$. The data is from the calculated curves in Fig. 3. The solid lines are numerical fits to the data which can be used to estimate the width of a lognormal distribution. The width of the distribution can be determined using $x=\sqrt{3} f_{V_{2}^{\prime} \text {,peak }} / f_{V_{2}^{\prime \prime}=0}: \sigma=$ $-\ln (x /(1.14-x)) / 3.09+0.67$ or using $x=-\min \left(V_{2}^{\prime \prime} / V_{\mathrm{AC}}\right) / \max \left(V_{2}^{\prime \prime} / V_{\mathrm{AC}}\right)$ : $\sigma=-\ln (x /(0.16-x)) / 3.00+0.49$

a lognormal distribution of Brownian relaxation frequencies,

$$
p_{\ln }^{\prime}\left(f_{\mathrm{B}}\right)=\frac{1}{f_{\mathrm{B}} \sigma_{f_{\mathrm{B}}} \sqrt{2 \pi}} \exp \left(\frac{-\ln ^{2}\left(f_{\mathrm{B}} / f_{\mathrm{Bm}}^{\prime}\right)}{2 \sigma_{f_{\mathrm{B}}}^{2}}\right),
$$

where $f_{\mathrm{Bm}}^{\prime}$ is the median Brownian relaxation frequency and $\sigma_{f_{\mathrm{B}}}$ is the width of the power-law-weighted distribution. The corresponding number-weighted lognormal distribution, $p_{\mathrm{In}}$, can be found by scaling the median as shown in Eq. (37). Then the number fraction of particles with Brownian relaxation frequencies between $f_{\mathrm{B}}$ and $f_{\mathrm{B}}+\mathrm{d} f_{\mathrm{B}}$ is $p_{\mathrm{ln}}\left(f_{\mathrm{B}}\right) \mathrm{d} f_{\mathrm{B}}$.

Figs. 3a-b and c-d show the real and imaginary parts of $\widetilde{\chi}=$ $\left(\chi^{\prime}-\mathrm{i} \chi^{\prime \prime}\right) / \chi_{0}$ (Eq. (19)) and $V_{2} / \widetilde{V}_{\mathrm{AC}}$ (Eq. (32)), respectively, calculated vs. $f / f_{\mathrm{Bm}}^{\prime}$.

Fig. 3b shows that $\chi^{\prime \prime}$ peaks at $f=f_{\mathrm{Bm}}^{\prime}$ irrespective of the width of the distribution. Therefore, the frequency corresponding to the peak in $\chi^{\prime \prime}$ can be used to extract the Brownian relaxation frequency of a particle with median size. For broad size distributions, it is observed that $\chi^{\prime} \neq \chi^{\prime \prime}$ at $f_{\mathrm{Bm}}^{\prime}$ and consequently, the frequency where $V_{2}^{\prime \prime}$ crosses zero, $f_{V_{2}^{\prime \prime}=0}$, is shifted to higher values as observed in Fig. 3d. Generally, it can be shown that

$$
f_{V_{2}^{\prime \prime}=0}=f_{\mathrm{Bm}}^{\prime} \exp \left(\sigma_{f_{\mathrm{B}}}^{2}\right) .
$$

When the width of the size distribution increases, the negative peak in $V_{2}^{\prime \prime} / \widetilde{V}_{\mathrm{AC}}$ just above $f / f_{\mathrm{Bm}}^{\prime}=1$ is smeared out and consequently for broad size distributions, $f_{V_{2}^{\prime \prime}=0}$ is not well-defined. Hence, for broad size distribution a different strategy is needed to determine the hydrodynamic size.

Fig. 3c shows that the peak position of $V_{2}^{\prime}, f_{V_{2}^{\prime}}$,peak , also depends on the width of the distribution, but to a much smaller extent than $f_{V_{2}^{\prime \prime}=0}$. This makes $f_{V_{2}^{\prime} \text {,peak }}$ a more robust estimator for the median Brownian relaxation frequency for disperse size distributions.

The width of the lognormal distribution can be estimated using the ratio between the positive and negative peak in $V_{2}^{\prime \prime}$ or from 
the ratio between $f_{V_{2}^{\prime} \text {,peak }}$ and $f_{V_{2}^{\prime \prime}=0}$ as shown in Fig 4 .

\section{Experimental}

\subsection{Materials}

Suspensions of particles with a remanent magnetic moment and nominal diameters of $80 \mathrm{~nm}$ (MM80: $80 \mathrm{~nm}$ BNF-Starch, 10-00801, Micromod, DE) and $100 \mathrm{~nm}$ (MM100: $100 \mathrm{~nm}$ BNF-Starch, 10-00-102, Micromod, DE) were diluted in de-ionized water to particle concentrations of $0.91 \mathrm{~g} / \mathrm{L}$ and $0.83 \mathrm{~g} / \mathrm{L}$, respectively, corresponding in both cases to an iron concentration of $0.5 \mathrm{~g} / \mathrm{L}$.

\subsection{Optomagnetic measurements}

Figure 1b shows a schematic of the readout system described previously by Donolato et al. ${ }^{14}$ In brief, a photodetector (ThorLabs PDA36A) collected light from a laser (Sanyo Blu-ray optical pickup, $\lambda=780 \mathrm{~nm}$ ) after the light passed through the measurement chamber on a microfluidic disk. The chamber had a thickness of $0.92 \mathrm{~mm}$, which defined the optical path length. Two electromagnets positioned along the optical axis produced a sinusoidally varying magnetic field resulting in a modulation of the light transmission through the MNP suspension. The signal from the photodetector, $V(t)$, was collected using a data acquisition card (National Instruments 6251). The frequency $f$ of the applied magnetic field was swept from $4 \mathrm{~Hz}$ to $2603.5 \mathrm{~Hz}$ in 38 logarithmically equidistant steps. A complete field and frequency sweep was recorded in less than $10 \mathrm{~min}$.

\subsection{AC susceptibility measurements}

Dynamic AC susceptibility (ACS) measurements were performed using the commercially available DynoMag ${ }^{\mathrm{TM}}$ AC susceptometer. ${ }^{22,23}$ Samples of $200 \mu \mathrm{L}$ were measured in the frequency range from $1 \mathrm{~Hz}$ up to $100 \mathrm{kHz}$ at room temperature. In the measurements, the amplitude of the excitation field started at $0.5 \mathrm{mT}$ at lower frequencies and falled off above $10 \mathrm{kHz}$. At $100 \mathrm{kHz}$, the amplitude was about $0.1 \mathrm{mT}$. The well predicted change in field amplitude was considered in the determination of the AC susceptibility. The ACS system was calibrated (with respect to amplitude and phase) in the whole frequency range. A measurement consisting of 30 points was recorded in $20 \mathrm{~min}$.

\subsection{Vibrating sample magnetometry}

Hysteresis loops were measured at room temperature in a LakeShore Model 7407 vibrating sample magnetometer (VSM). Samples were prepared by pipetting a volume of the MNP suspensions into thin-walled plastic tubes, capturing the MNPs at the bottom of the tubes, removing most of the supernatant and letting the remaining liquid evaporate over several days. The mass of each sample was determined as the difference in mass of the tube before the sample was added and after drying of the sample. The resulting sample pellet was fixed using transparent nail polish. The measured hysteresis loops and more details on the analysis are given in the Section $\mathrm{S}^{\dagger}$. The remanent magnetic moment of each of the particle samples was estimated assuming that the particles were non-interacting with randomly oriented uniaxial anisotropy axes. For this case, the measured remanent magnetic moment is equal to half the value of the average remanent magnetic moment of the particles.

\subsection{Dynamic light Scattering}

Dynamic light Scattering (DLS) measurements were performed using a Zetasizer Nano ZS. The samples were diluted to $0.25 \mathrm{~g} / 1$ and measured three times each. The mean Z-average and PDI were calculated from the measurements using the instrument software.

\section{Results and discussion}

Below, we first apply the equilibrium theory developed in Section 2.2 to determine the remanent magnetic moment of the particles for the two investigated MNP systems. Then, we apply the theory developed in Section 2.3 to determine the distribution of hydrodynamic sizes of the MNPs for the same two systems.

\subsection{Magnetic moment determination}

The magnetic moment distribution can be obtained from measurements of the optomagnetic signal at low frequency vs. the amplitude $B_{0}$ of the magnetic flux density. Fig. 5a shows the optomagnetic signal for the MM100 sample vs. time during a cycle in a $f=0.5 \mathrm{~Hz}$ sinusoidal magnetic field modulation for the indicated values of $B_{0}$ and where $B(t=0)=0$. First, we note that the photodetector signal and hence the intensity of transmitted light is lower when the magnetic field is low than when it is high, i.e., $\Delta \sigma=\sigma_{\|}-\sigma_{\perp}<0$. For this size range of particles, this observation indicates that the particles have a lower geometrical cross-section along the magnetic moment than perpendicular to it, which could be due to the particles being elongated along the magnetic moment. Next, we consider the dependence on $B_{0}$. At low values of $B_{0}$, a sine response of frequency $2 f$ is observed. At high values of $B_{0}$, a "peak-like" response is observed. This agrees well with the expectations from the equilibrium model, see Fig. 2a. No phase lag of the signal is observed with respect to the magnetic field. It is therefore justified to apply the equilibrium model described in Section 2.2. To proceed we calculate the 2nd harmonic optomagnetic signal, $V_{2}$.

Fig. 5b shows $V_{2}^{\prime \prime}$ for the MM80 and MM100 samples vs. $B_{0}$. For the MM100 sample, we observe a peak in $V_{2}^{\prime \prime}$ as expected for a large MNP magnetic moment. For the MM80 sample, this peak is not observed and thus a smaller MNP magnetic moment is expected.

To determine the particle magnetic moments for each of the two samples, the equilibrium model, Eq. (26), was fitted to the data. It was not possible to obtain a good fit assuming a single magnetic moment. Instead, the data were analyzed with a lognormal distribution of magnetic moments, Eq. (31), with $p_{\text {ln }}^{\prime}(m)=(m / \tilde{m})^{n_{\sigma} / n_{m}} p_{\ln }(m)$ (see Section 2.4.1). Free parameters in this fit were $n z \widetilde{V_{\text {ref }}} \Delta \widetilde{\sigma}, m_{\mathrm{m}}^{\prime}$ and $\sigma_{m}$. The value of $n_{\sigma} / n_{m}$ affects only the median value of the magnetic moment as described in Section 2.4.1 and the median value for $n_{\sigma} / n_{m} \neq 0$ can be calculated using Eq. (36). The fits for the two samples are shown as solid lines in Fig. 5b and the obtained magnetic moment distri- 

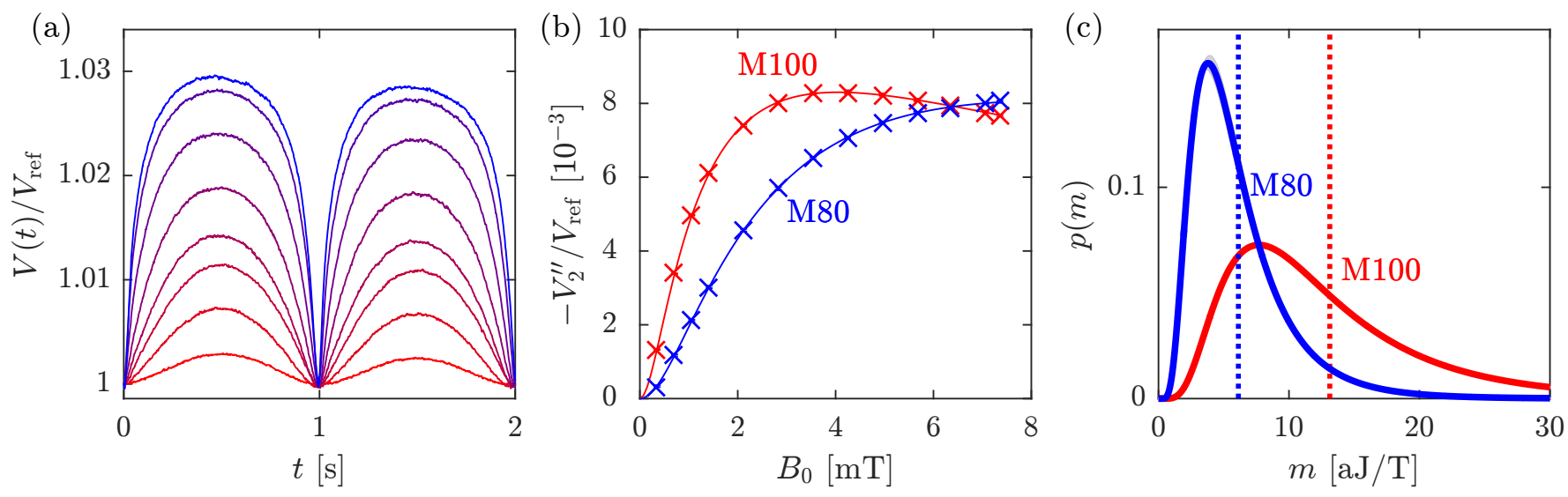

Fig. 5 (a) Photodetector signal measured vs. time for a $0.5 \mathrm{~Hz}$ sine excitation of the MM100 sample for $B_{0}=0.35,0.71,1.06,1.42,2.12,2.83,3.54$, 4.24, 4.95, 5.66, 6.37, 7.07 and $7.36 \mathrm{mT}$. (b) Measured 2nd harmonic imaginary signal at $f=0.5 \mathrm{~Hz}$ (points) for MM80 (blue) and MM100 (red) versus $B_{0}$. The solid lines are fits to the equilibrium model, Eq. (31), with $n_{\sigma} / n_{m}=0$ using a lognormal distribution of magnetic moments. (c) Lognormal magnetic moment distributions obtained from the fits in (b). The vertical dotted lines indicate the mean magnetic moments of $6.12 \mathrm{aJ} / \mathrm{T}$ and $13.13 \mathrm{aJ} / \mathrm{T}$ for the MM80 and MM100 samples, respectively.

butions are shown in Fig. $5 c$.

From the fits we obtain median magnetic moments $m_{\mathrm{m}}^{\prime}$ of $5.22 \mathrm{aJ} / \mathrm{T}(\mathrm{MM} 80)$ and $10.98 \mathrm{aJ} / \mathrm{T}$ (MM100) and lognormal distribution widths $\sigma_{m}$ of 0.57 (MM80) and 0.59 (MM100), respectively. Next, we estimate values of $n_{\sigma}$ and $n_{m}$ to establish the weighting of the signal in the above fits and to obtain the median value of the number-weighted lognormal distribution of magnetic moments, $p_{\ln }(m)$. Both investigated samples are multi-core MNPs with no extra non-magnetic shell $\left(t_{s} \simeq 0\right)$. We can therefore approximate $n_{m} \approx 1 / 2$. At present, the value of $n_{\sigma}$ is unknown, but assuming that absorption dominates the light extinction, the extinction is proportional to the particle volume, i.e., $n_{\sigma} \approx 1$. Then, according to Eq. (31), the lognormal distribution is scaled with $m$ to a power of $n_{\sigma} / n_{m} \approx 2$. The corresponding mean values of the $m^{2}$-weighted distribution calculated using Eq. (38) are given in Table 2. The number-weighted median magnetic moments are estimated from the above values of $m_{\mathrm{m}}^{\prime}$ and $\sigma_{m}$ using Eq. (36) to $3.2 \mathrm{aJ} / \mathrm{T}$ (MM80) and $6.4 \mathrm{aJ} / \mathrm{T}$ (MM100), respectively.

The weighting of the measurement magnetic moments obtained from VSM and ACS measurements is as follows: For a single MNP, the ACS signal is proportional to $m^{2}$. The remanent magnetic moment measured by VSM is weighted by the mass (or volume) of the particle. For the ideal multi-core particle, $m \propto V^{1 / 2}$ or $V \propto \mathrm{m}^{2}$, and hence the VSM measurement can also be considered to be $m^{2}$-weighted. Table 2 compares the obtained mean $m^{2}$ weighted magnetic moments from optomagnetic measurements to corresponding results obtained from VSM and ACS measurements (Sections S5-S6 ${ }^{\dagger}$ ).

The magnetic moments determined by ACS are 10-30\% higher than those determined using the OM method. Note, that the ACS determination of the magnetic moment from the initial susceptibility requires prior knowledge of the particle concentration and that these measurements are performed in a weak magnetic field, whereas the OM measurements do not rely on knowledge on the particle concentration and are performed as function of the magnetic field amplitude. The ACS measurements can be calibrated to a traceable standard whereas this is not easily feasible for the $\mathrm{OM}$ measurements due to the dependence on the anisotropy of the optical extinction properties of the particles. These factors as well as the simplifying assumption of $n_{\sigma} / n_{m} \approx 2$ may be the cause of the observed deviations between the two techniques. The magnitude of the remanent magnetic moment determined by VSM relies on the assumption of non-interacting particles as well as knowledge on the size and mass density of the MNPs. Magnetic dipole interaction between the MNPs are generally demagnetizing and this may cause an underestimation of the magnetic moment. Considering the differences between the techniques and the above mentioned possible sources of deviation, the agreement between the obtained values is remarkable.

Table 2 Mean $\mathrm{m}^{2}$-weighted magnetic moments obtained from optomagnetic measurements, the magnitude of the AC susceptibility data and from the remanent magnetic moment in VSM measurements.

\begin{tabular}{lll}
\hline Name & MM80 & MM100 \\
\hline $\bar{m}_{\text {OM }}[\mathrm{aJ} / \mathrm{T}]$ & $6.12(3)$ & $13.13(3)$ \\
$\bar{m}_{\text {ACS }[\mathrm{aJ} / \mathrm{T}]}$ & 8.2 & 14.7 \\
$\bar{m}_{\text {VSM }}[\mathrm{aJ} / \mathrm{T}]$ & 4.8 & 14 \\
\hline
\end{tabular}

\subsection{Size determination}

\subsubsection{Optomagnetic measurements}

The hydrodynamic size can be obtained from a measurement of $V_{2} / V_{\text {ref }}$ vs. frequency $f$ of a weak applied magnetic field. Figures 6a-b show $V_{2}^{\prime} / V_{\text {ref }}$ and $V_{2}^{\prime \prime} / V_{\text {ref }}$ vs. $f$ measured for $B_{0}=$ $0.25 \mathrm{mT}$.

First, we note that the signals are generally negative. A comparison to Fig. 3 reveals that $V_{\mathrm{AC}}$ and hence also $\Delta \sigma=\sigma_{\|}-\sigma_{\perp}$ is negative (see Eq. (25)) in agreement with the observation for the equilibrium response in Section 4.1.

From the Brownian relaxation frequency, the hydrodynamic di- 
ameter of an equivalent sphere can be obtained from Eq. (1) as

$$
\left.D_{\mathrm{h}}=\sqrt[3]{k_{\mathrm{B}} T /\left(\pi^{2} \eta f_{\mathrm{B}}\right.}\right),
$$

where $T=22^{\circ} \mathrm{C}$ and $\eta=9.548 \cdot 10^{-4}$ Pa s.

Before we estimate values of the Brownian relaxation frequency, it is relevant to discuss how the OM signal is weighted with the hydrodynamic volume, which is proportional to $f_{\mathrm{B}}^{-1}$. In Eq. (37) we found that the signal is weighted by $f_{\mathrm{B}}^{-\left(n_{\sigma}+2 n_{m}\right) / n_{\mathrm{h}}}$. Using the previously estimated values of $n_{\sigma} \approx 1$ and $n_{m} \approx 0.5$ and noting that the hydrodynamic volume is proportional to the particle core volume $\left(n_{\mathrm{h}}=1\right)$, we have $\left(n_{\sigma}+2 n_{m}\right) / n_{\mathrm{h}} \approx 2$, i.e., the OM signal is weighted by the square of the hydrodynamic volume.

The analysis presented below was performed using Eq. (32) with the volume-squared $\left(V_{\mathrm{h}}^{2}\right)$-weighted lognormal distribution, $p_{\ln }^{\prime}\left(f_{\mathrm{B}}\right)=\left(f_{\mathrm{B}} / \tilde{f}_{\mathrm{B}}\right)^{-\left(n_{\sigma}+2 n_{m}\right) / \sigma_{n}} p_{\ln }\left(f_{\mathrm{B}}\right)=\left(f_{\mathrm{B}} / \tilde{f}_{\mathrm{B}}\right)^{-2} p_{\ln }\left(f_{\mathrm{B}}\right)$, and resulted in the median value, $f_{\mathrm{Bm}}^{\prime}$, of the Brownian relaxation frequency and a lognormal standard deviation $\sigma_{f_{\mathrm{B}}}$.

The number-weighted distribution of Brownian relaxation frequencies, $p_{\ln }\left(f_{\mathrm{B}}\right)$, is also a lognormal distribution with the same value of $\sigma_{f_{\mathrm{B}}}$ and a median value given by Eq. (37) with $\left(n_{\sigma}+\right.$ $\left.2 n_{m}\right) / n_{\mathrm{h}} \approx 2$.

The volume $\left(V_{\mathrm{h}}\right)$-weighted distribution of Brownian relaxation frequencies, which will also be of interest below, has the same width and a median value of $f_{\mathrm{Bm}} \approx f_{\mathrm{Bm}}^{\prime} \exp \left(\sigma_{f_{\mathrm{B}}}^{2}\right)$. The volumeweighted median hydrodynamic diameter $D_{\mathrm{m}}$ can be obtained by insertion of $f_{\mathrm{Bm}}$ in Eq. (41). Further, we note that $\sigma_{D_{\mathrm{h}}}=\sigma_{f_{\mathrm{B}}} / 3$.

First, we consider the peak in the $V_{2}^{\prime}$ signal. As described in Section 2.4.1 the position $f_{V_{2}^{\prime} \text {,peak }}$ of this peak is only weakly sensitive to a lognormal distribution of hydrodynamic sizes, and it relates to the median Brownian relaxation frequency as $f_{V_{2}^{\prime} \text {,peak }} \approx$ $f_{\mathrm{Bm}}^{\prime} / \sqrt{3}$. From the data in Fig. 6 a we obtain values of $f_{V_{2}^{\prime \prime}=0}$ (marked with circles) of $120 \mathrm{~Hz}$ (MM80) and $75 \mathrm{~Hz}$ (MM100), respectively, corresponding to $V_{\mathrm{h}}^{2}$-weighted median hydrodynamic diameters $D_{V_{2}^{\prime} \text {,peak }}^{\prime}$ of $128 \mathrm{~nm}$ (MM80) and $149 \mathrm{~nm}$ (MM100), respectively.

Next, we consider the $V_{2}^{\prime \prime}$ signal, which shows a sign change at a frequency $f_{V_{2}^{\prime \prime}=0}$ and which for the two investigated samples displays a negative saturation value at low frequencies and a positive peak just above $f_{V_{2}^{\prime \prime}=0}$. For a monodisperse sample, we found in Section 2.3 that $f_{\mathrm{B}}=f_{V_{2}^{\prime \prime}=0}$. For a polydisperse sample, we found in Section 2.4.1 that $f_{V_{2}^{\prime \prime}=0}$ is significantly shifted to higher values and therefore that the value of $f_{V_{2}^{\prime \prime}=0}$ does not represent the median Brownian relaxation frequency, $f_{\mathrm{Bm}}^{\prime}$. Further, the ratio between $\sqrt{3} f_{V_{2}^{\prime} \text {,peak }}$ and $f_{V_{2}^{\prime \prime}=0}$ could be used to estimate the value of $\sigma_{f_{\mathrm{B}}}$. From the data in Fig. 6b we obtain values of $f_{V_{2}^{\prime \prime}=0}$ (marked with large points) of $553 \mathrm{~Hz}$ (MM80) and $269 \mathrm{~Hz}$ (MM100), respectively. Combining with Fig. 4, we estimate $\sigma_{f_{\mathrm{B}}}=0.90$ (MM80) and $\sigma_{f_{\mathrm{B}}}=0.77$ (MM100). In Section 2.4.1 we also found that $\sigma_{f_{\mathrm{B}}}$ could be estimated from the ratio between the absolute values of the minimum and maximum values (the ratio between the valley and hill) of the $V_{2}^{\prime \prime}$ spectrum as shown in Fig. 4. From the data in Fig. 6b, we obtain $\sigma_{f_{\mathrm{B}}}=0.91$ (MM80) and $\sigma_{f_{\mathrm{B}}}=0.70$ (MM100), respectively. The estimates of $\sigma_{f_{\mathrm{B}}}$ using these two methods are consistent. Knowing $\sigma_{f_{\mathrm{B}}}$ and $f_{V_{2}^{\prime \prime}=0}$, we can use Eq. (40) to estimate the median $V_{\mathrm{h}}^{2}$-weighted Brownian relaxation frequency
$f_{\mathrm{Bm}}^{\prime}$ and hence the median $V_{\mathrm{h}}^{2}$-weighted hydrodynamic diameters $D_{V_{2}^{\prime \prime}=0}^{\prime}$, which are found to be $121 \mathrm{~nm}$ (MM80) and $138 \mathrm{~nm}$ (MM100), respectively.

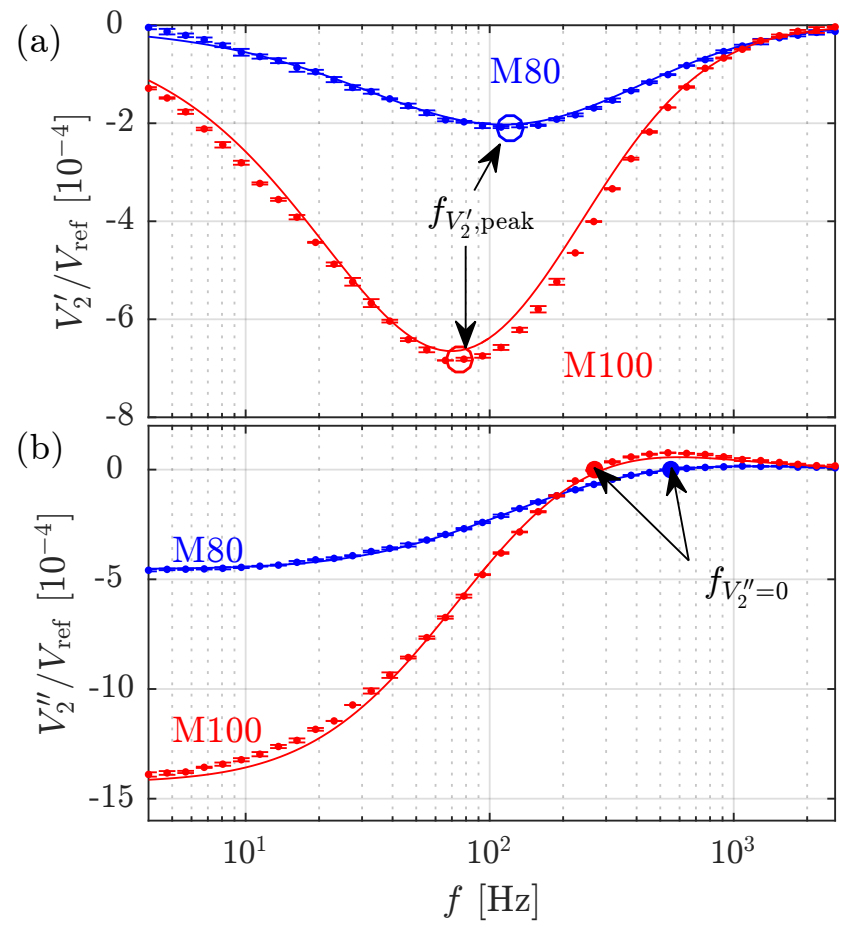

Fig. 6 (a) Real and (b) imaginary parts of the second harmonic optomagnetic signal measured vs. frequency at $B_{0}=0.25 \mathrm{mT}$ for the MM80 sample (blue) and the MM100 sample (red). The solid lines are fits of Eq. (32) to the data with a lognormal distribution of Brownian relaxation frequencies, Eq. (39).

Finally, we also analyzed the measured optomagnetic spectra in terms of Eq. (32) assuming a constant value of $\tilde{V}_{\text {AC. }}$ As previously, the fit was performed with a $V_{\mathrm{h}}^{2}$-weighted lognormal distribution of Brownian relaxation frequencies given as $p_{\ln }^{\prime}\left(f_{\mathrm{B}}\right)=$ $\left(f_{\mathrm{B}} / \tilde{f}_{\mathrm{B}}\right)^{-2} p_{\ln }\left(f_{\mathrm{B}}\right)$ with the median hydrodynamic diameter, $f_{\mathrm{Bm}}^{\prime}$, and width $\sigma_{f_{\mathrm{B}}}$. The fits resulted in $V_{\mathrm{h}}^{2}$-weighted median hydrodynamic diameters $D_{\mathrm{h}}^{\prime}$ of $122 \mathrm{~nm}$ (MM80) and $145 \mathrm{~nm}$ (MM100) and widths $\sigma_{f_{\mathrm{B}}}$ of 0.93 (MM80) and 0.84 (MM100), respectively.

Comparing the median sizes obtained from the fits to the values obtained by the zero crossing and peak position, we observe a good correlation between the methods for MM80. The value obtained from the peak position is slightly larger because the peak position moves to lower frequency for a broad size distribution as observed in Fig. 3c.

The fit for MM100 does not fully describe the data. This indicates that distribution is not lognormal or that the distribution is too wide for the assumptions to be valid, e.g., that the simple power law assumption with a constant $n_{\sigma}$ for the size dependence of $\sigma_{\|}$and $\sigma_{\perp}$ is not fulfilled for all particle sizes.

The results of the optomagnetic measurements in terms of the volume-weighted median, which will be useful for comparison with other methods as we will describe below, are summarized in Table 3. 
Table 3 Median volume-weighted hydrodynamic particle diameters and standard deviations $\sigma_{D_{\mathrm{h}}}$ of their lognormal distribution. For the optomagnetic (OM) measurements, values were obtained from analyses of $f_{V_{2}^{\prime \prime}=0}$, $f_{V_{2}^{\prime} \text {,peak }}$ and a full curve fit, respectively. For DLS the value was obtained as the Z-average from the instrument software. For the ACS measurements, values were obtained from the peak position of $\chi^{\prime \prime}$ and a full curve fit, respectively.

\begin{tabular}{llll}
\hline Tech. & Name & MM80 & MM100 \\
\hline OM & $D_{V_{2}^{\prime \prime}=0}[\mathrm{~nm}]\left(\sigma_{D_{\mathrm{h}}}\right)^{a}$ & $92(0.30)$ & $117(0.23)$ \\
OM & $D_{V_{2}^{\prime}, \text { peak }}[\mathrm{nm}]\left(\sigma_{D_{\mathrm{h}}}\right)^{b}$ & $97(0.30)$ & $123(0.26)$ \\
OM & $D_{V_{2}, \text { fit }}[\mathrm{nm}]\left(\sigma_{D_{\mathrm{h}}}, \text { PDI }\right)^{c}$ & $92(0.31,0.10)$ & $114(0.28,0.08)$ \\
ACS & $D_{\chi_{2}^{\prime \prime} \text {,peak }}[\mathrm{nm}]^{d}$ & 92 & 117 \\
ACS & $D_{\chi, \text { fit }}[\mathrm{nm}]\left(\sigma_{D_{\mathrm{h}}}, \text { PDI }\right)^{e}$ & $95(0.29,0.09)$ & $116(0.21,0.04)$ \\
DLS & $D_{z}[\mathrm{~nm}](\mathrm{PDI})^{f}$ & $99(0.08)$ & $121(0.09)$ \\
\hline
\end{tabular}

$a$ The median sizes obtained from the zero crossing of $V_{2}^{\prime \prime}$ and the distribution widths, $\sigma_{D_{\mathrm{h}}}$, estimated using the ratio between the maximum and minimum value of $V_{2}^{\prime \prime}$.

$b$ Sizes obtained from the peak position of $V_{2}^{\prime}$ and distribution widths, $\sigma$, estimated from $f_{V_{2}^{\prime} \text {,peak }} / f_{V_{2}^{\prime \prime}=0}$.

$c$ Median, sigma value $\left(\sigma_{D_{\mathrm{h}}}\right)$ and polydispersity index (PDI) obtained from a fit to OM data with a lognormal distribution.

$d$ Sizes obtained from the peak position of $\chi^{\prime \prime}$

$e$ Median, sigma value $\left(\sigma_{D_{\mathrm{h}}}\right.$ ) and polydispersity index (PDI) obtained from a fit to ACS data with a lognormal distribution.

$f Z$-average obtained from DLS measurements.

\subsubsection{Comparison to DLS and ACS measurements}

Below we compare the sizes obtained using the optomagnetic method with those obtained using ACS and DLS. For this comparison, it is important that the data are obtained using the same weighting with the particle size. Therefore, we also need to estimate the weighting of the reference methods.

In ACS measurements, the signal is weighted by the magnetic moment squared, as shown in Eq. (19). For multi-core particles, such as the MM80 and MM100, the magnetic moment is expected to be proportional to the square root of the particle volume, $m \propto$ $V^{1 / 2}\left(n_{m} \approx 0.5\right)$. Thus, $V \propto m^{2}$ and we expect the ACS result to be volume-weighted.

Table 3 compares the results obtained using the optomagnetic method (volume-weighted), with sizes determined from ACS measurements (Section $\mathrm{S}^{\dagger}$ ). In the ACS measurements, the hydrodynamic size was estimated either using the peak position, $D_{\chi_{2}^{\prime \prime} \text {,peak }}$, or from a fit to the Debye model using a lognormal distribution of sizes, $D_{\chi}$,fit, as described by Öisjöen et al. ${ }^{24}$ (details are given in Section $\mathrm{S6}^{\dagger}$ ). It should be noted that this analysis does not require knowledge of the particle concentration. It should further be noted that ACS studies the magnetic relaxation, which may include both Brownian and Néel relaxation contributions, whereas the optomagnetic technique directly probes the magnetic-field-induced change in the physical orientation of the particles and thus is only indirectly sensitive to Néel relaxation via a possible induced magnetic moment and magnetic shape anisotropy of the particles. Finally, the optomagnetic technique additionally depends on the anisotropy of the optical extinction properties of the particles.

We observe an excellent agreement between the ACS results and the optomagnetic results for the MM80 sample. For the
MM100 sample, the values of the median hydrodynamic sizes are in good agreement, but the width of the hydrodynamic size distribution is slightly smaller using ACS measurements than using OM measurements.

In DLS measurements, the signal is weighted by the scattering properties of the particle (at $90^{\circ}$ in the present study). For small particles with $D \ll \lambda$, the particle size dependence is described by Rayleigh scattering, which is proportional to the volume square. This results in the Z-value to be weighted by $D_{\mathrm{h}}^{5 / 6}$. However, for larger particles, as in our case, the scattering becomes angle dependent and no simple size dependence exists. However, comparing the Z-values to the mean volume-weighted sizes obtained from ACS measurements ( $99 \mathrm{~nm}$ and $119 \mathrm{~nm}$ ) and OM measurements (96 nm $119 \mathrm{~nm}$ ) calculated using Eq. (38), we observe an excellent agreement. This suggests that the DLS signal is also approximately volume-weighted.

In summary, we have found that it is important to account for the different dependencies of the signals on the particle to be able to obtain comparable values between the different methods. However, when this dependence was accounted for, the agreement between the employed methods is remarkable, especially considering that the optomagnetic signal depends on a combination of magnetic and optical properties of the particles. The detailed dependence of, e.g., the magnetic moment and of the scattering properties of the investigated multi-core particles are still not known in detail and therefore the method is at present restricted to be applicable to particle ensembles with narrow size distributions. For broader size distributions, the method may still be applied but it is not quantitative. In particular the optomagnetic method is very sensitive to larger magnetic particles or aggregates of particles, which is advantageous for detection of agglomerates of particles in biosensing applications. Further, due to its simplicity, speed and low requirement for the knowledge of other sample parameters, such as the particle concentration, the optomagnetic method can be highly useful for quality monitoring in the production of magnetic nanoparticles with a remanent magnetic moment. Finally, we note that the optomagnetic method is at present the only method to determine the relation/correlation between magnetic and optical anisotropies for an ensemble of particles.

\section{Conclusion}

We presented the theory for the optomagnetic signal in equilibrium as function of the magnitude of the applied oscillating magnetic field and have derived how this response can be used to deduce the remanent magnetic moment of magnetic nanoparticles in suspension. Further, we presented the theory for the dynamic optomagnetic response as function of frequency at low magnetic fields and how dynamic optomagnetic measurements can be used to extract the hydrodynamic size of magnetic nanoparticles in suspension. Finally, we carefully considered the influence of a distribution of particle properties on the above results and provided quantitative relations describing the influence of lognormal size distributions on the characteristic features of the dynamic optomagnetic response.

The presented theory was applied for the determination of the 
remanent magnetic moment and the hydrodynamic size of two commercially available multi-core magnetic nanoparticle systems and the results were compared to those obtained using vibrating sample magnetometry, AC susceptibility measurements and dynamic light scattering. The comparison illustrated the importance of accounting for the weighting of the signal in the different techniques, which will affect the median value of a lognormal distribution of a parameter but not the width of the distribution. In dynamic light scattering and AC susceptibility measurements, the signals are weighted by the optical and magnetic properties, respectively, whereas in optomagnetic measurements, both the magnetic moment and the optical extinction properties influence the signal. When the weighting of the signals with the particle size was appropriately accounted for, the results obtained by the different techniques were found to be in good agreement.

The presented optomagnetic technique has several advantages compared to the other applied techniques: It can be realized using low-cost components, it is compact and flexible as it requires only a transparent sample container, and a complete measurement can be performed in less than 10 min. Further, it provides information on both the remanent magnetic moment and the hydrodynamic size of the magnetic nanoparticles without requiring prior knowledge on the particle concentration. Finally, it provides unique information on the link between the remanent magnetic moment and the optical anisotropy of the particles.

On the downside, the technique is limited to nanoparticles with a remanent magnetic moment and with linked optical and magnetic anisotropies and cannot at present be referred to traceable standards. Moreover, it requires that the size distribution of the nanoparticle cores is comparatively narrow such that the particles have similar extinction properties.

We believe that the presented method provides a simple approach to characterize suspensions of magnetic nanoparticles with a remanent magnetic moment and a narrow size distribution and thus that it is attractive for, e.g., routine monitoring of the quality of such suspensions in a production or laboratory setting.

\section{Acknowledgment}

The authors would like to thank Miriam Varón, Department of Physics, Technical University of Denmark for help in performing DLS measurements. This work is financially supported by EU FP7 grant No. 604448-NanoMag.

\section{References}

1 Q. A. Pankhurst, J. Connolly, S. K. Jones and J. Dobson, Journal of Physics D: Applied Physics, 2003, 36, R167.

2 F. Varenne, A. Makky, M. Gaucher-Delmas, F. Violleau and C. Vauthier, Pharm. Res., 2016, 33, 1220-1234.

3 A. Agbabiaka, M. Wiltfong and C. Park, J. Nanoparticles, 2013, 2013, 640436.

4 F. Ahrentorp, A. Astalan, J. Blomgren, C. Jonasson, E. Wetterskog, P. Svedlindh, A. Lak, F. Ludwig, L. J. van IJzendoorn, F. Westphal, C. Grüttner, N. Gehrke, S. Gustafsson, E. Olsson and C. Johansson, J. Magn. Magn. Mater., 2015, 380, 221226.

5 F. Ludwig, A. Guillaume, M. Schilling, N. Frickel and A. M. Schmidt, J. Appl. Phys., 2010, 108, 033918.

6 S. Bogren, A. Fornara, F. Ludwig, M. del Puerto Morales, U. Steinhoff, M. Hansen, O. Kazakova and C. Johansson, Int. J. Mol. Sci., 2015, 16, 20308-20325.

7 A. Lak, A. F. Thünemann, M. Schilling and F. Ludwig, J. Magn. Magn. Mater., 2015, 380, 140-143.

8 J. Dieckhoff, D. Eberbeck, M. Schilling and F. Ludwig, J. Appl. Phys., 2016, 119, 043903.

9 A. Ranzoni, G. Sabatte, L. J. van IJzendoorn and M. W. J. Prins, ACS Nano, 2012, 6, 3134-3141.

10 S. Schrittwieser, F. Ludwig, J. Dieckhoff, K. Soulantica, G. Viau, L.-M. Lacroix, S. M. Lentijo, R. Boubekri, J. Maynadié, A. Huetten, H. Brueckl and J. Schotter, ACS Nano, 2012, 6, 791-801.

11 S. Schrittwieser, F. Ludwig, J. Dieckhoff, A. Tschöpe, A. Günther, M. Richter, A. Huetten, H. Brückl and J. Schotter, Small, 2013, 10, 407-411.

12 M. Donolato, P. Antunes, T. Z. G. de la Torre, E.-T. Hwu, C.-H. Chen, R. Burger, G. Rizzi, F. G. Bosco, M. Strømme, A. Boisen and M. F. Hansen, Biosens. Bioelectron., 2015, 67, 649-655.

13 R. S. Bejhed, T. Z. G. de la Torre, M. Donolato, M. F. Hansen, P. Svedlindh and M. Strömberg, Biosens. Bioelectron., 2015, 66, 405-411.

14 M. Donolato, P. Antunes, R. S. Bejhed, T. Zardán Gómez de la Torre, F. W. Østerberg, M. Strömberg, M. Nilsson, M. Strømme, P. Svedlindh, M. F. Hansen and P. Vavassori, Anal. Chem., 2015, 87, 1622-1629.

15 A. Mezger, J. Fock, P. Antunes, F. W. Østerberg, A. Boisen, M. Nilsson, M. F. Hansen, A. Ahlford and M. Donolato, ACS Nano, 2015, 9, 7374-7382.

16 B. Tian, R. S. Bejhed, P. Svedlindh and M. Strömberg, Biosens. Bioelectron., 2016, 77, 32-39.

17 A. Günther, P. Bender and R. Birringer, J. Phys.: Condens. Matter, 2011, 23, 325103.

18 M. Gratz and A. Tschöpe, J. Phys. D. Appl. Phys., 2017, 50, 15001.

19 T. Klein, A. Laptev, A. Günther, P. Bender, A. Tschöpe and R. Birringer, J. Appl. Phys., 2009, 106, 114301.

20 F. Ludwig, O. Kazakova, L. F. Barquín, A. Fornara, L. Trahms, U. Steinhoff, P. Svedlindh, E. Wetterskog, Q. A. Pankhurst, P. Southern, P. Morales, M. F. Hansen, C. Frandsen, E. Olsson, S. Gustafsson, N. Gehrke, K. Lüdtke-Buzug, C. Grüttner, C. Jonasson and C. Johansson, IEEE Transactions on Magnetics, 2014, 50, 1-4.

21 P. W. Barber and D.-S. Wang, Appl. Opt., 1978, 17, 797.

22 A. Prieto Astalan, C. Jonasson, K. Petersson, J. Blomgren, D. Ilver, A. Krozer and C. Johansson, J. Magn. Magn. Mater., 2007, 311, 166-170.

23 T. Zardán Gómez de la Torre, A. Mezger, D. Herthnek, C. Johansson, P. Svedlindh, M. Nilsson and M. Strømme, Biosens. Bioelectron., 2011, 29, 195-199. 
24 F. Öisjöen, J. F. Schneiderman, A. P. Astalan, A. Kalabukhov, C. Johansson and D. Winkler, Biosens. Bioelectron., 2010, 25, 1008-1013. 